
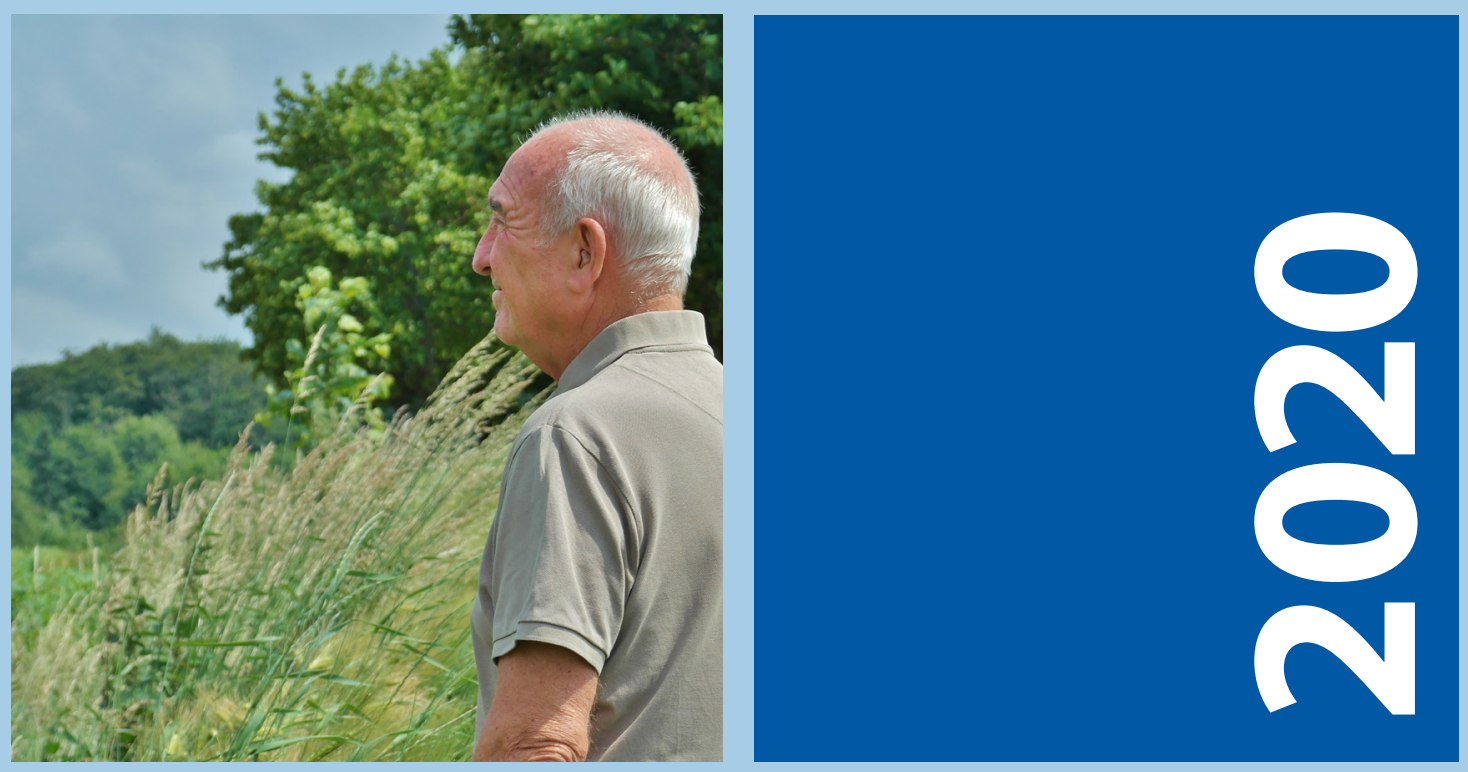

\title{
TILDA Nursing Home Data: A short report to inform COVID-19 responses for our most vulnerable
}

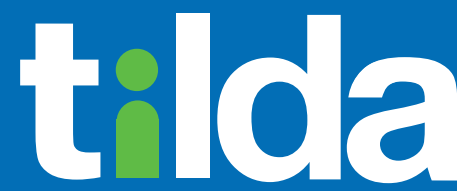

Staidéar Fadaimseartha na hÉireann um Dhul in Aois

The Irish Longitudinal Study on Ageing 


\section{TILDA Nursing Home Data: A short report to inform COVID-19 responses for our most vulnerable}

Roman Romero-Ortuno, Peter May, Minjuan Wang, Siobhan Scarlett, Ann Hever, Rose Anne Kenny

On behalf of the TILDA team

May 2020 
Copyright (C) The Irish Longitudinal Study on Ageing 2020

The Irish Longitudinal Study on Ageing

Trinity College Dublin

Dublin 2

Tel: +35318962509

Email: tilda@tcd.ie

Website: www.tilda.ie

ISBN: 978-1-907894-32-9

https://www.doi.org/10.38018/TildaRe.2020-08 


\section{Acknowledgements}

We would like to acknowledge the vision and commitment of our study funders, the Department of Health, the Health Research Board, Science Foundation Ireland, The Atlantic Philanthropies, and Irish Life plc. We would like to state that any views expressed in this report are not necessarily those of the Department of Health or of the Minister for Health. We would also like to thank the TILDA participants without whom this research would not be possible. 


\section{Introduction}

The Irish Longitudinal Study on Ageing (TILDA) was originally designed to study the health and socioeconomic circumstances of community-dwelling people aged 50 or more in Ireland. The study began in 2009 and over 8,500 people took part in the first wave of the study in 2009 and 2010. TILDA will soon be collecting data for its sixth wave (2020-2021) (https://tilda.tcd.ie/about/where-are-we-now/). Over this 10-year period, some participants originally included as community-dwelling transitioned to a nursing home, and some last participant interviews were conducted in this setting. In addition, TILDA has collected information on deceased participants through the end-of-life interviews completed by family members and friends. From the latter, a small number of participants were reported to have died in a nursing home. In this short report we describe the characteristics of participants from those two data sources, which are not mutually exclusive. 


\section{Nursing Home Interviews}

Of the 100 participants who were interviewed in a nursing home, the mean (SD) age was 83.7 (7.2) years and 62\% were female. Eighty-five had a long-term health problem, illness or disability limiting their day-to-day activities, and 26 had a history of dementia or cognitive problem.

When asked to self-rate their physical health, 61 could not self-report due to a cognitive and/or physical problem; 22 reported that their physical health was 'good, very good or excellent', and 12 reported it as 'fair'. The remaining 5 reported their physical health as 'poor'.

When asked to self-rate their emotional/mental health, 61 could not self-report; 26 reported that their emotional/mental health was 'good, very good or excellent', and 11 reported it as 'fair'. The remaining 2 reported it as 'poor'.

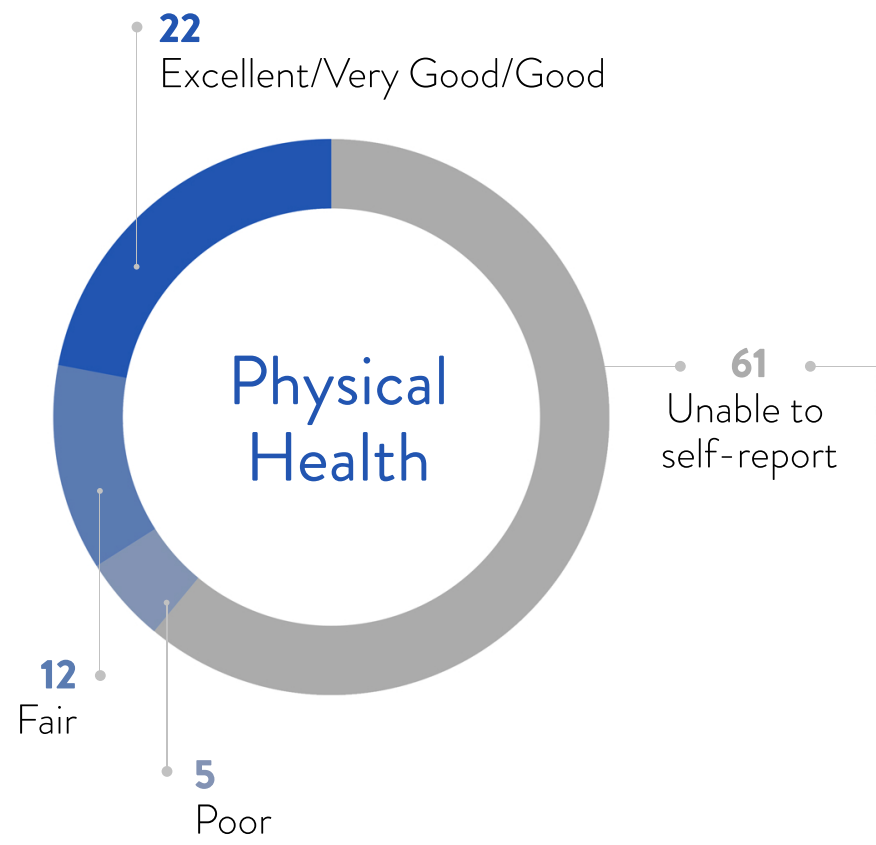
Excellent/Very Good/Good 


\section{End-of-life interviews}

To date, TILDA has collected 718 interviews on the end-of-life experience of deceased participants. Among these, 97 deaths occurred in a nursing home; the mean (SD) age at death was $85.0(8.5)$ years and $51(53 \%)$ were female.

Fifty-eight percent had a history of hypertension, $46 \%$ of Alzheimer's and related dementias, $31 \%$ congestive heart failure, $20 \%$ diabetes, $17 \%$ heart attack, $17 \%$ cancer, $13 \%$ anxiety and $11 \%$ depression.

Eighty percent had had disability for 4 or more basic activities of daily living (ADLs), and a further $13 \%$ had had disability in 1-3 basic ADLs. Seventy-nine percent had had disability for 4 or more instrumental ADLs, and a further 10\% had had disability in 1-3 independent ADLs.

Fifty-seven percent had been troubled by pain in the last year of life, and $61 \%$ had fallen in the last year of life.

The most common causes of death were cardiovascular/circulatory (32\%), respiratory (16\%), infection (13\%) and cancer (10\%). 


\section{Conclusions}

In this small descriptive series, TILDA nursing home participants were chronologically very old, had very high levels of physical and cognitive morbidities, and very high levels of physical disability. Indeed, nursing homes look after the most vulnerable in society.

Despite the above, when TILDA nursing home participants were able to self-report, a majority reported that their physical and mental health was fair, good, very good or even excellent. Not being able to self-report was mostly associated with the presence of cognitive and communication problems, including dementia.

The personal perspectives of our TILDA nursing home participants provide an important reminder that quality of life is often rated higher by oneself than by proxies, even in the presence of very advanced age and extensive comorbidities and disabilities.

From these two data sources alone, it is not possible to infer the proportion or incidence of institutionalisation in the Irish population. The small number of participants included in this short report comes from a secondary data analysis and is not necessarily representative of the nursing home population in Ireland.

With the above caveats in mind, in this short report we have described the characteristics of TILDA's small sample of nursing home participants. TILDA cares very much about our nursing homes residents. Nursing home residents have been disproportionately affected by the COVID-19 pandemic and we will welcome further research that includes this very vulnerable group. 